\title{
Relaciones literarias gallego-catalanas (1920-1939): Materiales y propuestas para su estudio
}

\author{
JUAN M. RIBERA LLOPIS \\ Universidad Complatense de Madrid \\ Olivia Roukíguez GoNZÁlez \\ limiversidad de La Coruña
}

\section{JUSTIFICACIÓN}

Dentro del programa de los XI Cursos de Verano de la UNED y en el Centro Asociado de Pontevedra (3-7 de julio de 2000) se impartió el curso Castelas) y su tiempo, algunas de cuyas comunicaciones se recogen en el presente volumen de $R L L C G V$ como el lector fácilmente poxlrá reconocer por sus títulos y contenidos, aunque aparezcan redistribuidas en las respectivas secciones de artículos y no en el lógico apartado de actas del que esta publicación parecía disponer. Con ocasión de dicho curso, el primer firmante de estas páginas propuso, en primer lugar y mediante datos precisos, una aproximación hacia el proceso que, desde finales del ochocientos, afianzó el encuentro gallego-catalán que se coronaría ya entrado el novecientos; y, en segundo lugar y más consideradamente, se detuvo en tres calas, estas sí del tiempo de Castelao, que merecían un comentario menos sincrético. Hoy, ya conjuntamente quienes venimos trabajando hace tiempo en esa materia, hemos pensado en la oportunidad de ordenar todos esos referentes histórico-literarios en unos materiales y casi a modo de fic has que, aquí presentados, acaso puedan sugerir nuevas lecturas, apreciaciones o juicios a partir de la nolicia de su existencia y contrastados con otros documentos y datos desconocidos por nosotros. No es otra la pretensión de estas páginas. Partimos por tanto de trabajos previos y firmados en colaboración en los que datos, títulos y personalidades citados ya han merecido 
la reconsideración tenida por oportuna en el momento de su redacción. lo cual, es cierto, no ha impedido seguir hallando nuevos ángulos de lectura y encontrando nuevos factores y pruebas de aquella relación histórica. Un par de trabajos individuales por parte del primer firmante, y ya remotos, sólo cobran valor en la medida en que nos pusieron a ambos en la pista que hoy recorremos juntos y que se cifra en las siguientes aportaciones:

- Relacions entre Vicente Risco e Jom Maragall, Actas do (ongreso Vicente Risco (Ourense, 1995), Santiago de Compostela, Xunta de Galicia, 1995, pp. 331-351.

- Aproximación ás relacions literarias galego-catalanas. Noticias histiricas e hibliograficas, Madrygal, UCM, n"1. 1998, pp. 97-100.

- A imaxe e o concepto do "galego" nas literaturas galega, castelá e" catalana da fin do século XIX, Actas do V Congreso Internacional de' Estudios Galegos (Trier, 1997), Trier, Ediciós do Castro-Galicien Zentrum der Universität Trier, 1999, vol. I, pp. 73-83.

- Joan Salvat-Papasseit e Manuel Antonio: O mar como espacio poético da aventura. Cinguidos por unha arela comun. Homenaxe of Profesor $X$. Alonso Montero, eds. R. Álvarez, D. Vilavedra, Santiago de Compostela, Universidade, 1999, vol. II, pp. 1261-1273.

- Crisi finisecular en la cultura gallega: atenció al model català, Actes de Congrés Internacional 1898: Entre la crisi d'identitat i la modernització (Barcelona, 1998), Barcelona, Publicacions de l'Abadia de Montserrat, 2000, vol. I, pp. 249-262.

- O Modernismo do "Cenáculo de Ourense" e a atención os modelos catalans, Actas del Congreso Internarional Literatura Modernista y Tiempo del 98 (Lugo 1988), Santiago de Compostela. Universidade, 2000, pp. $529-543$.

La síntesis seguidamente propuesta nos ubica en el encuentro moderno de dos tradiciones peninsulares, la de aquellos sendos romances, "catalân de navegantes, galaico de labradores..." poetizados por Valle-Inclăn, que, entre los siglos XIX y XX, buscaron trazar un arco «... del Cabo de Creus al de Finisterre», según coordenada de Aurelio Ribalta y bajo expectatjvas regeneracionistas. Todo ello, a favor de una «relación dialéctica de influencia mutua» (A. Magariños, 1979: 40), en la cual, cabría reconocer, hubo más constancia y devoción desde las márgenes atlánticas. La atención. hoy, a aquellos intentos y logros, interesa mutuamente a ambos espectros de cara a conocer sus sendas historias. Quizá en función de aquel criterio se pueda justificar que trabajemos prioritariamente desde documentación gallega: también y tal vez en función de ello se pueda argüir que el corpus generado desde Galicia fue mayor. 


\section{ANTECEDENTES}

Cabe señalar una cronología que se consolida de la década de los ochenta del siglo XIX a bien entrada la segunda década del siglo XX. Una cronología que, para el caso catalán, se traza entre fechas emblemáticas como 1881, aparición de LAvens, y 1917, muerte de Enric Prat de la Riba; y para el caso gallego, entre 1885, muerte de Rosalía y fundación de la «Biblioteca Gallega» de Andrés Martínez Salazar, y 1915. fundación de las «Irmandades da Fala». Entre esos referentes, otras correspondencias que posiblemente forjaron un espectro paralelo fueron las siguientes:

1887: Lliga de Catalunya - 1891: Asociación Regionalista Gallega. 1897: Liga Gallega.

1906: Solidaritat Catalana - 1907: Solidaridad Gallega.

Ante esas correspondencias puede ordenarse toda una documentación que, alendiendo a su contenido, quedaría en dos apartados: uno primero, en el que se desarrolla un discurso socio-político que, acorde con las fechas, progresa de la ideologia regionalista a la iberista y que en ocasiones remite a la actividad literaria (a) lo cual, de ser así, señalaremos; y otro segundo, que se centra en la vida literaria (b):

(a)

- M. Murguía, Los prectursores (1886), con noticias sobre la Renaixenşa y sobre la recepción literaria de Rosalía.

- M. Murguia, Galicia (1888, ed. en Barcelona).

- A. Brañas, El Regionalismo (1889, ed. en Barcelona), contiene aproximación a las letras catalanas, de la Edad Media al siglo XIX; recoge polémica coetánea en torno a la participación de obras sólo en castellano o no en el premio de teatro de la RAE, finalmente conseguido por $F$. Soler con Batalla de Reynas.

- M. Murguía, Mestre de lo (atay Saber en los Joes Florals de Barcelona (1890), con lectura de discurso (ed. O. Rodríguez, 2000).

- A. Brañas, Bases generales del regionalismo (1892), texto con incidencia en las posteriores Bases de Mamresa.

-- A. Brañas y F. Pi i Margall, doble conferencia complementaria en Santiago de Compostela (1892).

- A. Brañas, discurso en los Jocs Florals de Barcelona (1893), leído por $\mathrm{J}$. Verdaguer y en el que se contemplan los símiles Cataluña-Polonia y Galicia-Irlanda.

- A. Ribalta, Catalanismo militante (1901), contiene aproximación a las letras catalanas desde B.C. Aribau a V. Balaguer. 
- E. López. Aydillo, Galicia ante la Solidaridad (1907), libro-encuesta con referencias a Calaluña y, entre los entrevistados, un catalín.

- En A Nosa Torra (fund. 1907) articulos de y sobre Cataluña como «Viva España!» de J. Maragall.

- E. Carré, Influencia de los catalanes en el progreso de la industria pesquera de Galicia (1908), leído ya en la Real Acadèmia de Bones Lletres (Barcelona) en 1903 y publicado en su Butlletí.

- Artículos de J. Maragall entre 1893 y 1897 . sobre postulados regionalistas de A. Brañas (v. O.C.).

- A. Ribalta, «Liga de Amigos del Idioma» en Estudios Gallegos (1916. $\mathrm{n}^{\circ} 17$ ).

(b)

- d. 1868: Recepción de Rosalía (v. C. Hermida Gulias, 1993).

- años 80: V. Balaguer, colaboración en Xogos Frorais Galegos y discurso de recepción en la RAE, Las literaturas regionales (1883).

-- 1890: Homenaje gallego por ed. de El Regionalismo de A. Brañas; en A Monteira (Lugo, $n^{\circ} 22$ ), mensajes de adhesión de J. Rubió i Ors, A. Guimerà y N. Oller.

- 1891: Traslado de los restos de Rosalía al Panteón de Bonaval; con este motivo, poemas de J. Rubió i Ors y T. Llorente en La Parria Gallega (Santiago). Entre estas fechas, poemas de J. Rubió i Ors en Galicia (La Coruña) y artículos de la prensa catalana y seguimiento de la literatura catalana en Revista Gallega (La Coruña).

- 1892: M. Curros Enríquez, Aires de mi tierra, ed. valenciana en castellano traducida por el renaixencista C. l.lombart y prologada por V. Blasco Ibáñez, estableciendo símiles con la función de J. Verdaguer.

- 1893: M. Milà i Fontanals: no sólo realiza estudios sobre lírica gallega medieval y lírica popular de Galicia, sino que se manifiesta sobre la complicidad y el reconocimiento para con el Rexurdimento (v. O.C., vol. V). Edición políglota de Lo Gayter del Llobregat (1888-1992, 4 vols.) de J. Rubió i Ors, con traducción al gallego de A. Brañas (vol. de 1893).

- 1898: G. Rodríguez Salinas, La dramática gallega (Revista gallega, II, n" 87), a propósito del teatro regional y de un factible teatro gallego. menciona el teatro satírico valenciano y la escena catalana de F. Soler a A. Guimerà y V. Balaguer, traducido y representado en casteflano, en una verdadera comprensión del teatro español.

- 1903: Carta de J. Maragall a J. Pijoan relatando el viaje a La Coruña, mediante nociones y registro coincidente con su poética, levantando 
una imagen modernista continuada en otros artículos castellanos sobre una Galicia literaturizada (v. O.C.).

- 1905: Pretacio de J. Maragall a Poesia \& Prosa de l. Rivera i Rovira eon referencias al atlantismo literario y al calma" gallega por él conocida a través de Rosalía y de Curros.

- 1906: Estètica arbitrària de G. Alomar, posiblemente recogida por V. Risco en La filosofia arbitraria (El Miño, 1909-1910); así como, si no la noción, sí la voz de efuturisme» de Alomar (1904), a través de una conferencia del catalán en la Universidad de Madrid (1909), pucto alcanzar a V. Risco en fiuturismo (E/ Miño).

- años 10: Atención del «Cenáculo de Ourense» y de otros modernistas gallegos, así A. Rey Soto en Divagaciones en torno a la poesia (1915). a la literatura modernista catalana, proyectada desde Barcelona o divulgada desde Madrid (sucesivamente en La España Regional, La lectura, La Gaceta Literaria...). Lecturas margallianas de V. Risco. asumiendo las propuestas de la Teoria de la paraula viva para su interpretación De Literatura Gallega (Mi Tierra. Orense, junio y julio, 1911 ) y aludiendo directamente en Preludio a toda estética fistura (La Centuria, Orense, junio-julio, 1917); y gradual atención a E. d'Ors y al «arbitrarisme» que desarrollaría el referente indicado en 1906 . Volvemos en el apartado siguiente sobre la relación Risco-Ors. A propósito de esas relaciones en años de formación. véase el recuento de $V$. Risco en Nós, os inadaptados (Nós, 1930) que a Maragall y Ors añade el conocimiento de Diego/Didac Ruiz., cuyas tesis en tomo al esoterismo $y$ at "acte entusiasta» incidirían en el orensano. Risco volveria sobre sus maestros catalanes en El centenario do Maragall y Más sobre Maragall (La Región, Orense, 1960, n¹5767 y 15773).

\section{TRES CALAS.}

a. A propósito de A.R. Castelao (1886-1956): Teniendo presente el volumen de X.L. García (1988), Castelao dende Catalunva, con las críticas que no obstante se le pudicran hacer por la no reordenación y revisión de todo un material precedente, cabe señalar que las alusiones catalanas en sus obras aparecen a partir de 1912; entre esa fecha y 1918 habría que situar una no confirmada exposición de sus dibujos en el Ateneu barcelones: y relacionados con la presencia y el protagonismo político de Castelao en el eje Cataluña-Galicia-País Vasco - de la Triple Alianza (1923) a la fundación de Galeuza (1933)- eslarán sus sucesivos viajes a Barcelona desde 1932, este junto con R. Otero Pedrayo. hasta 1937. Este año llega a Barcelona desde Valencia. ciudades entre las cuales comienza a escribir Sempre en Galiza. 
Por lógica, la actividad de Castelao en geografía catalana en liempos de crisis prebélica o en pleno contlicto civil está marcada por intereses políticos que limitan lo que hubiera podido ser un intercambio literario y artístico más preciso. No obstante, preguntamos y sugerimos, contando con el substrato inmediato de los años diez y de perfiles modernistas, si no hay una relación entre su obra plástica y ciertos modelos y prácticas catalanes. Si cabe la hipótesis de que las alegorías pre-rafaclitas de S. Rusiñol, los ambientes mágicos de los carteles de A. Gual y los grabados de A. de Riquer o el medievalismo y las fantasías plásticas de A. Mestres, sean contemplados desde grabados de $L a$ Centuria, así el alegórico ex-libris de V. Risco en el $n^{\circ} 3$ de esa publicación, ipor qué no mirar sobre cierta correspondencia entre parte de la iconografía de I. Nonell y la de Castelao? iy si cerrásemos el triángulo con el noruego $\mathrm{E}$. Munch, pensando en sus grabados?

b. A propósito de la poesía de la vangzardia: El triángulo de inicial referencia lo cierran en esta ocasión V. Risco - T. Garcès - J. Salvat Papasseit. Entre cllos se dedican mutuamente poemas y libros; valgan como muestras los poemas de Risco a los catalanes en $A$ Nosa Terra (enero, 1919). Risco, además, extractará y comentará el manifiesto de Salval. Contra els poetes amb minúscula en $A$ Nosa Terra (v. X.R. Pena, 1996: 75), con comentarios añadidos de Garcès, y, en la misma publicación y en 1920 , reseñará volúmenes poéticos de J. Salvat Papasseit y de J.M. de Junoy. A este espectro, la figura incorporada es la de Manoel Antonio, cuya correspondencia revela que fue Risco quien le orientó en el terreno de las vanguardias, animándole a escribir un "manifiesto» y a lograr un poeta "étnico» equivalente al "poeta cívic» aclamado por los catalanes: en carta de 1920 (v. M. Antonio, 1979: 74-79). V. Risco le dice al poeta que piensa enviarle junto a su Preludio a toda estética futura, el manifiesto de Salvat y le nombra el de V. Huidobro. Así, M. Antonio tiene a finales de año el manifiesto catalán que incidirá en la gestación del propio Mais alá! (1922) firmado junto con A. Cebreiro.

Lo que debería contemplarse, ya que sucle citarse ctéreamente hermanados a J. Salvat Papasseit y M. Antonio, es confrontar con más precisión sus poéticas. En trabajo referido hemos atendido al mar como espacio poético de la arentura presente en ambos vanguardistas. Vitalismo y posibles correspondencias iconográficas aproximan sus dos forjas marítimas, mientras que la contemplación urdidora de fantasías frente a la experiencia directa del mar, respectivamente, puede diferenciarlos. ¿Quién indagará en ese y otros recovecos de los dos poetas, frente a frente?

c. A propósito de la relación entre Vicente Risco y Eugeni d'Ors: A lo largo de los años veinte $V$. Risco mantiene su atención por J. Maragall al tiempo que añade, como referente catalán, el nombre de S. Rusiñol. En Os europeos en Abramtes (1927) se cita El Poble Gris (1902), al tiempo que del texto catalán sobre el gallego se proyectan la estructura mediante escenas costumbristas 
fragmentadas y la óptica bumorístico-deformadora de personajes-tipo. También resonancias del medio rusiñoliano puede haber en la constitución por Risco de la "Orde Galega do Santo Graal". donde resuena "La Santa Confraria»" fundada en las Festes Modernisters de Sitges.

Entre ese entramado va surgiendo el influjo de E. d'Ors, divulgado en castellano y peninsularmente mediante la traducción de G. Martínez Sierra y la resultante La muerte de Lsidro Nonell y orras arbitrariedades (1905). V. Risco será uno de sus difusores desde sus artículos de El Miño, junto con otros nombres como E. Montes, reconociéndose estos dos como discípulos del demiurgo catalán. Señalemos como reflejo en Risco de aquel Ors, su Oración a Madona Frivolidad (El Miño, 1909-1910) cuyo pre-rafaelitismo se mira en el de Oración a Madona Blanca María de la colectánea orsiana cilada. Pero cabe pensar que, gradualmente, «o filosofo catalán» irá interesando a Risco más como pensador que como creador literario. De ese recorrido y creciente atención al Ors programador, según la tesis doctoral (UCM. 1992) de O. Rodríguez pendiente de convertirse en un proyecto editorial de Galaxia, se deduce que el Novecentismo risquiano difundido desde La Cemturia pudo haberse construido sobre presupuestos orsianos, aunque acabara por apuntar hacia direcciones propias, el gallego. como por cjemplo es su defensa del siglo XIX. También pudiera haberse sustentado Risco en la teoria orsiana de las tres civilizaciones para forjar su teoría del "Atlantismo" expuesta en Teoria do Nacionalismo Galego (1920), aunque con ella acabara por vislumbrar la función reconductora y futura de la "Civilización Allántica» sobre un "europeísmo mediterráneo" que se equiparaba con la ucrisis del europeísmo". Por Jo que aquí venimos tratando. cabe leer en ese punto de llegada una conciencia de madure $z$ por parte del escritor y de la cultura galaica que venía a neutralizar las diferencias de prioridad detectables en el tándem Cataluña-Galicia que, con ciertos desajustes, había llevado un ritmo cultural de correspondencias en aquel cruce de siglos. Se cerraba así un ciclo histórico cuya esencia habría de relanzarse mediante diferentes estrategias tras la crisis bélica y social de los años treinta del novecientos y a través de la situación política de los años cuarenta a los sesenta. Todo un recorrido, el de aquel ciclo, que pudiera dibujarse de los poemas gallegos de C. Riba de 1911 (ed. X. Alonso Montero, 1961) a los catalanes de A. Cunqueiro de 1935 (ed. X. Alonso Montero y G. Avenoza, 1998). Ante aquel. quizás sólo cupiese la rememoración, como la que le dedicara A. Calvet i Pasqual "Gaziel» en La Península imakahada (1961). De lo que podemos estar seguros es que más documentación y nuevas relecturas de lo hasta ahora ordenado están a la espera de recuperarse y de replantearse para eslablecer mejor el horizonte de aquellas relaciones literarias. 


\section{REFERENCIAS BIBLIOGRÁFICAS}

Alonso Montero, X., ed. (1993): Carles Riba e Galicia, Vigo, Galaxia.

Ai.onso Montero, X.. Avenozza i Vera, G., eds. (1998): Dous pocmas en calalán de Álvaro Cunqueiro, Madrygal. Resisza de Estudios Gallegos. UCM, n" 1, pp. 27-37. Garcis, X.L. (1988): Castelao dende Catalunva, Sada - A Coruña, Ediciós do Castro. Hermida Gulias, C. (1993): Rosalía de Castro na prensa barcelomesa (1863-1899), s.l., Edicións do Patronato Rosalía de Castro.

MAgiariños, A. (1979): Los gallegos, Barcelona, Epidauro.

Mantel Antonio (1979): Carrespondencia III, ca. de D. Garcia Sabell, Vigo. Galaxia. Pt:NA. X.R. (1996): Manuel Antonio e a vangarda, Santiago de Compostela, Sotelo Blanco.

RODRÍliUEZ GONZALLZ, O., ed. (2000): Recuperación dun discurso esquecido de Manuel Murguía, Madrygal Revista de Estudios Gallegos, UCM, n’3, pp. 93-96. 